\title{
A COVID-19 Decision Support System for Phone Call Triage, Designed by and for Medical Students
}

\author{
Jean-Patrick VREL ${ }^{\mathrm{a}, \mathrm{b}, 1,2}$, Samy OULMANE ${ }^{\mathrm{a}, \mathrm{b}, 2}$, Adrien BOUKOBZA ${ }^{\mathrm{a}, \mathrm{b}}$, \\ Anita BURGUN ${ }^{\mathrm{b}, \mathrm{c}}$ and Rosy TSOPRA ${ }^{\mathrm{b}, \mathrm{c}}$ \\ a Université de Paris, Faculté de médecine, Paris, France \\ ${ }^{\mathrm{b}}$ Department of Medical Informatics, Hôpital Européen Georges-Pompidou, AP-HP, \\ Paris, France \\ ' INSERM, Université de Paris, Sorbonne Université, Centre de Recherche des \\ Cordeliers, Information Sciences to support Personalized Medicine, F-75006 Paris,
} France

\begin{abstract}
During spring 2020, SARS-CoV-2 pandemic induced shortage of medical equipment, hospital capacity and staff. To tackle this issue, medical students have been strongly involved in early patient triage through medical phone call regulation. Here, we present an intelligent web-based decision support system for COVID-19 phone call regulation, developed by and for, medical students to help them during this difficult but crucial task. The system is divided into 5 tabs. The first tab displays administrative information, clinical data related to life-threatening emergency, and personalized recommendations for patient management. The second tab displays a PDF report summarizing the clinical situation; the third tab displays the guidelines used for establishing the recommendations, and the fourth tab displays the overall algorithm in the form of a decision tree. The fifth tab provided a short user guide. The system was assessed by 21 medical staff. More than $90 \%$ of them appreciated the navigation and the interface, and found the content relevant. $90,5 \%$ of them would like to use it during the medical regulation. In the future, we plan to use this system during simulation-based medical learning for the initial medical training of medical students.
\end{abstract}

Keywords. Clinical Decision Support System, Digital Health, Emergency, COVID19, Medical Simulation, Medical Education

\section{Introduction}

The SARS-CoV-2 pandemic has put national health systems under great pressure. All countries around the world had to face a shortage of medical equipment, hospital capacity and staff. Better management of these resources is becoming crucial and remains a challenge worldwide. Early patient triage through medical phone calls represents a key point to mitigate in-patient flow. An efficient and prompt triage method is required to better assign available resources and avoid time-wasting in patient management.

\footnotetext{
${ }^{1}$ Corresponding Author, Dr Rosy TSOPRA, Université de Paris, Faculté de médecine, Paris, France; E-mail: rosy.tsopra@nhs.net

${ }^{2}$ Co-first authors - These two authors contributed equally to this work
} 
To facilitate patient triage, algorithms have been developed [1], and a few of them have been computerized [2,3]. They could assist: (i) medical staff at hospital admissions [4], (ii) GPs during face-to-face or virtual visits [2], or (iii) patients through online tools. However, not much have been developed for professionals involved in the medical phone call regulation. Phone call regulation usually involved health care workers, but during the pandemic, medical students were also strongly involved, and may have met some difficulties to assess patients remotely. Textual documentations have been published, to guide them, such as the COVID-19 phone script published by the Centers for Disease Control and Prevention.

Here, we aim at developing an intelligent web-based decision support system for patient triage during phone call regulation, specifically devoted to medical students. The system was designed and assessed by and for medical students, during a new module training integrated during medical school curriculum at the University of Paris, named "Artificial intelligence for clinical decision support".

\section{Methods}

We developed an intelligent web-based decision support system to regulate patients' calls during medical phone call regulation. It proposes adaptative guidance for COVID19 patients based on patients' symptoms, and up-to-date recommendations.

\subsection{Design of the decision support system}

The decision tree was built from the French guidelines developed by SAMU 92 (Service d'Aide Médicale Urgente des Hauts de Seine) and other national sources. From these guidelines, all terms related to patient orientation were extracted and grouped into categories of variables. Then, we distinguished action and decision variables. Decision variables were made unambiguous and encoded as binary: true and false representing respectively the presence or absence of a sign. After that, we made all possible combinations with the decision variables. For each combination, we associated an action variable corresponding to the recommendations of the system for patient triage. These combinations covering all possible situations, constituted the rules base of the system. This rules base was then directly implemented into the system.

The interface was built according to the workflow of the medical phone regulation, but also ergonomic criteria for minimizing the "user-interface" interaction [5].

The system was implemented using RStudio version 1.3.1093, Shiny package. The decision tree algorithm was generated and used as a rpart object from the Rpart package.

\subsection{Evaluation of the clinical decision support system}

Medical students and other health staff were invited to evaluate the system. After a short presentation of the system, they had to test the system online by their own, through 1 to 3 clinical cases simulating a medical regulation. Then, they filled an anonymous online form to rate the system and give feedbacks about their experience. Questions were focused on aesthetics, utilization (2 questions), relevance (2 questions), and adoption (1 question). Each item was assessed through a Likert-scale [6,7]. Other elements could be added in free-text comment. 


\section{Results}

\subsection{Covid-19 decision support system for phone call triage}

The system displays 5 tabs (Figure 1, area a). The first tab allows the medical student to fill information about: administrative (area b), quick clinical assessment (area c), and advanced clinical assessment (area d). The information displayed is interactive and only showed when necessary, e.g., pregnancy for women, warning popups for life threatening situations. Likewise, the information displayed in the "advanced clinical assessment" area depends on the information filled in the "quick clinical assessment" area. Tooltips can also be used to help to fill the elements, and to prompt recommendations (area f). Once the medical student has filled all the information needed, then the system automatically generates a recommendation (area e).

The second tab displays a PDF report summarizing the clinical situation and the proposed recommendation. This report can be transmitted to senior medical experts present on the health care center.

The third tab displays the guidelines used for designing the system, and the fourth tab displays the overall algorithm in the form of a decision tree. A short user guide can be found in the fifth tab.

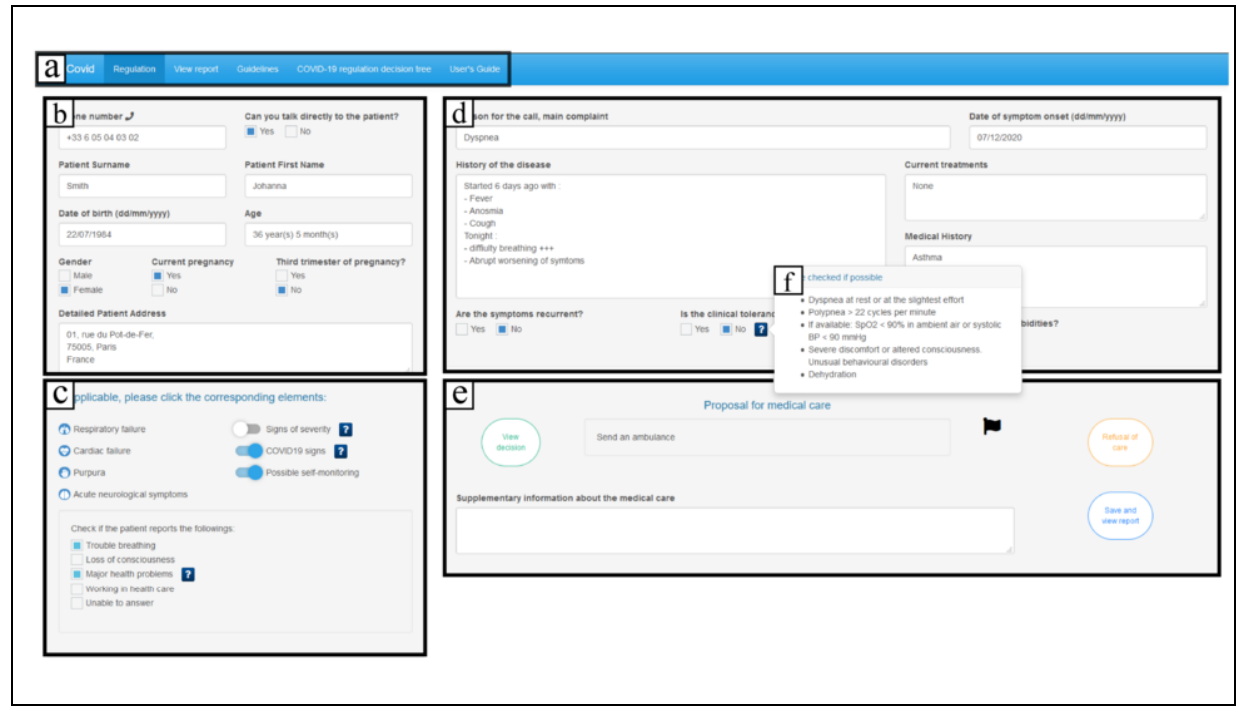

Figure 1. Clinical Decision Support System interface is divided into 5 tabs (area a). Here is shown the first one where the regulation is carried on. The user fills administrative information about the patient in area $b$ and indicates if there is a life-threatening emergency in areas $\mathrm{c}$ and $\mathrm{d}$. Then, a recommendation is displayed into area e. Tooltips are also available for helping to fill patient information (area f). 


\subsection{Evaluation of the clinical decision support system}

21 medical staff filled the survey (Figure 2$)$. Nineteen were medical students $\left(2\right.$ in $4^{\text {th }}$ year, 11 in $5^{\text {th }}$ year and 6 in $6^{\text {th }}$ year); one was a junior doctor, and one was a nurse. One third of them already had an experience in phone call regulation.

Regarding the user interface experience, $95.2 \%$ of participants found the interface simple and intuitive to use and all found it aesthetically pleasing. About the medical content of the system, $90.5 \%$ of participants found the requested information relevant, and all of them found the suggested guidance relevant to the clinical situation.

Finally, $90.5 \%$ of participant would like to use this system for COVID-19 patients' regulation.

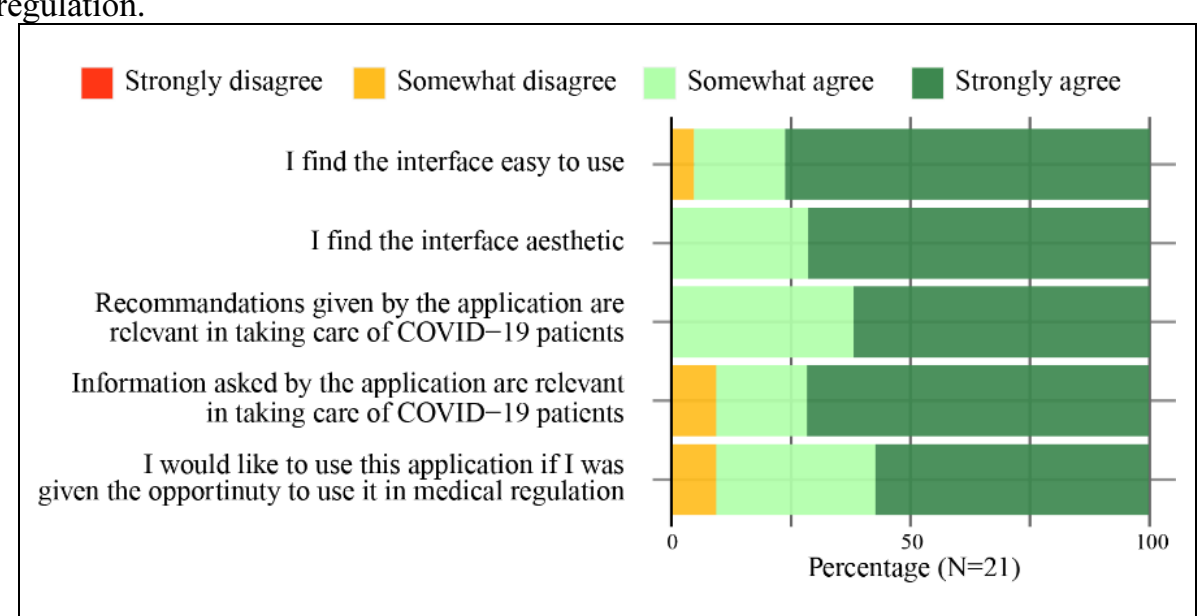

Figure 2. Summary of the results of the system evaluation.

\section{Discussion}

We developed an intelligent web-based system that proposes adaptative guidance for COVID-19 patients, based on patients' symptoms and up-to-date recommendations. The system was designed by and for medical students, in order to meet their needs and overcome their lack of expertise. Conversely to black box CDSS, our system is based on a decision tree which guarantees both explainability and transparency in decision making. The system was found pleasant, relevant and useful for the majority of evaluators.

Our work presents some limits. First, we do not consider speech recognition that would be very useful during phone call regulation. This could facilitate the filling of information, by extracting keywords from patient's speech and thus prevent cognitive tunneling during the regulation. In the future, we plan to implement a real-time speech detection, using deep learning algorithm framework. We also plan to improve the usability of the interface, e.g., by adding more meaningful colors and icons. Second, the system focuses only on the management of COVID-19 patients and doesn't cover all situations that could be met during medical regulation. In the future, we aim at extending the system to other critical situations. Third, the system is not yet implemented into a phone regulation center, and for that, further works are needed.

Other approaches have been designed for emergency call triage, such as the provision of algorithms, flowcharts or guidelines to health professionals [8,9]. They 
allowed to improve triage level accuracy [9], but may require a time for appropriation. Here we expect a better appropriation of the decision support system because it is incorporated into the clinical workflow, and the interactivity with health professionals is increased. Other approaches based on machine learning have also been described [10]. They may have good performance on triage, but are still limited to specific emergencies such as cardiac arrest [10]. In the future, we aim at designing a hybrid system using both knowledge issue from guidelines, and machine learning techniques.

Our system will be used during simulation-based medical learning [11]. Indeed, this system could be very useful during the initial medical training of medical students, because they could train by themselves with the system, and then have feedbacks from senior medical experts. In the future, we plan to implement the system in our University and to assess its effects on students learning.

\section{Funding}

This work was part of a new module taught during medical school curriculum at the University of Paris, named "Artificial intelligence for clinical decision support".

\section{References}

[1] Wallace DW, Burleson SL, Heimann MA, Crosby JC, Swanson J, Gibson CB, et al. An adapted emergency department triage algorithm for the COVID-19 pandemic. Journal of the American College of Emergency Physicians Open. 2020 Dec;1(6):1374-9.

[2] Liu Y, Wang Z, Ren J, Tian Y, Zhou M, Zhou T, et al. A COVID-19 Risk Assessment Decision Support System for General Practitioners: Design and Development Study. J Med Internet Res. 2020 Jun 29;22(6):e19786.

[3] McRae MP, Dapkins IP, Sharif I, Anderman J, Fenyo D, Sinokrot O, et al. Managing COVID-19 With a Clinical Decision Support Tool in a Community Health Network: Algorithm Development and Validation. J Med Internet Res. 2020 Aug 24;22(8):e22033.

[4] Wu G, Yang P, Xie Y, Woodruff HC, Rao X, Guiot J, et al. Development of a clinical decision support system for severity risk prediction and triage of COVID-19 patients at hospital admission: an international multicentre study. Eur Respir J [Internet]. 2020 Aug 20 [cited 2021 Jan 6];56(2). Available from: https://www.ncbi.nlm.nih.gov/pmc/articles/PMC7331655/

[5] Shneiderman B, Plaisant C. Designing the user interface: strategies for effective human-computer interaction. 5th ed. Boston: Addison-Wesley; 2010. 606 p.

[6] Marcolino MS, Oliveira JAQ, Cimini CCR, Maia JX, Pinto VSOA, Sá TQV, et al. Development and Implementation of a Decision Support System to Improve Control of Hypertension and Diabetes in a Resource-Constrained Area in Brazil: Mixed Methods Study. J Med Internet Res. 2021 Jan 11;23(1):e18872.

[7] Tsopra R, Courtine M, Sedki K, Eap D, Cabal M, Cohen S, et al. AntibioGame®: a serious game for teaching medical students about antibiotic use. International Journal of Medical Informatics. 2020 Jan $3 ; 104074$.

[8] Morreel S, Philips H, Colliers A, Verhoeven V. Performance of a new guideline for telephone triage in out-of-hours services in Belgium: A pilot study using simulated patients. Health Serv Manage Res. 2020 Nov;33(4):166-71.

[9] Brasseur E, Servotte J-C, Donneau A-F, Stipulante S, d'Orio V, Ghuysen A. Triage for out-of-hours primary care calls: a reliability study of a new French-language algorithm, the SALOMON rule. Scand J Prim Health Care. 2019 Apr 29;37(2):227-32.

[10] Blomberg SN, Folke F, Ersbøll AK, Christensen HC, Torp-Pedersen C, Sayre MR, et al. Machine learning as a supportive tool to recognize cardiac arrest in emergency calls. Resuscitation. 2019 May;138:322-9.

[11] Al-Elq AH. Simulation-based medical teaching and learning. J Family Community Med. 2010 Jan;17(1):35-40. 